\title{
On the Tapping Mode Measurement for Young's Modulus of Nanocrystalline Metal Coatings
}

\author{
H. S. Tanvir Ahmed, Eric Brannigan, and Alan F. Jankowski \\ Edward E. Whitacre, Jr. College of Engineering, Mechanical Engineering, Texas Tech University, P. O. Box 41021, \\ Lubbock, TX 79409-1021, USA \\ Correspondence should be addressed to Alan F. Jankowski; alan.jankowski@ttu.edu
}

Received 7 June 2013; Revised 26 July 2013; Accepted 27 July 2013

Academic Editor: Carlos R. Cabrera

Copyright (C) 2013 H. S. Tanvir Ahmed et al. This is an open access article distributed under the Creative Commons Attribution License, which permits unrestricted use, distribution, and reproduction in any medium, provided the original work is properly cited.

\begin{abstract}
Young's modulus of nanocrystalline metal coatings is measured using the oscillating, that is, tapping, mode of a cantilever with a diamond tip. The resonant frequency of the cantilever changes when the diamond tip comes in contact with a sample surface. A Hertz-contact-based model is further developed using higher-order terms in a Taylor series expansion to determine a relationship between the reduced elastic modulus and the shift in the resonant frequency of the cantilever during elastic contact between the diamond tip and sample surface. The tapping mode technique can be used to accurately determine Young's modulus that corresponds with the crystalline orientation of the sample surface as demonstrated for nanocrystalline nickel, vanadium, and tantalum coatings.
\end{abstract}

\section{Introduction}

A variety of indentation-based test methods are used to evaluate strengthening effects [1-4] in materials at the nanoscale. Nanoindentation normal to the surface is routinely used to measure the hardness and Young's modulus. Triboindentation tests are used [5-7] to measure both hardness and shear strength as well as quantify strain-rate sensitivity $[8,9]$ effects in the evaluation of deformation mechanisms in nanocrystalline alloys. The standard approach [10-12] to determine the elastic modulus during nanoindentation evaluates the load $(P)$ versus displacement $(h)$ curve during unloading after plastic deformation. It remains difficult to collect a sufficient quantity of $P$ versus $h$ data during elastic loading since elastic displacements $(z)$ are commonly limited to depths of only a few nanometers or less in many hard materials. The initial linear slope $(S)$ of the power-law-shaped unloading curve is used [11, 12] to determine the reduced elastic modulus $\left(E^{*}\right)$ as

$$
E^{*}=\frac{S \sqrt{\pi}}{2 \beta \sqrt{A_{c}}} .
$$

The contact area $\left(A_{c}\right)$ equals the square of the contact depth $\left(h_{c}^{2}\right)$ multiplied by the tip area $\left(c_{h}\right)$ coefficient, and the shape parameter $\beta$ equals 1.00 for flat punch, 1.034 for Berkovich, and 1.012 for Vickers indenter tips. The reduced elastic modulus $\left(E^{*}\right)$ of the indenter tip and sample surface system is related to the elastic moduli of the indenter tip and sample surface as

$$
\frac{1}{E^{*}}=\frac{1-v_{t}^{2}}{E_{t}}+\frac{1-v_{s}^{2}}{E_{s}}
$$

Here, the subscripts $t$ and $s$ represent the probe tip and sample, respectively, for the Poisson ratio $(\nu)$ and Young's modulus $(E)$ values. Indentation size effects (ISEs) are found with the directional loading of the indenter tip. For example, beyond an indentation depth, that is, $10 \%$ of the film thickness, the use of a Meyer plot indicates [13, 14] that the substrate material contributes to the elastic and plastic property measurements of the coating. Also, the sensitivity of the nanoindentation measurement to accurately measure anisotropic elastic behavior is shown [15-17] to be a function of the indenter tip shape. For example, a triangular indenter can give values that are much higher than axisymmetric tips such as spheroconical, but with less sensitivity than that obtained using axisymmetric shapes. Nanoindentation measurements with Berkovich diamond 
tips may not quantify the full effect of anisotropic behavior; that is, the anisotropic response can be underestimated in both modeling and experimental efforts. For example, a 10\% difference from 125 to $140 \mathrm{GPa}$ is reported [15] between $\mathrm{Cu}(100)$ and $\mathrm{Cu}(111)$, whereas the known elastic moduli $E(h k l)$ along different crystalline directions [ $h k l]$ vary by $300 \%$ with values from 66 to $192 \mathrm{GPa}$. Similarly, different effects are found $[18,19]$ for the effect of tip shape on plastic flow wherein spheroconical shapes evidence work hardening of material during indentation as opposed to well-defined pyramidal indentations that produce a near-perfect plastic response.

The torsion-resonance mode of atomic force acoustic microscope is used [20] to measure the elastic constants of anisotropic materials. A piezoelectric device is excited using an alternating current voltage to induce vibrations in the atomic force microscopy (AFM) cantilever while the tip is in contact with the sample surface. Indentation elastic modulus is extracted from the tip-surface interaction assuming Hertzian contact mechanics. In a similar technique [21], the deflection of the AFM cantilever is used to determine the localized modulus. Vibrating reed measurements [22] have similarities to the AFM technique where the major difference is that the sample along with the substrate in the vibrating reed method is exposed to piezoelectric vibrations whereas the probe cantilever is vibrated in the AFM technique. The oscillating bubble method [23] is another technique for measuring surface elasticity of liquids. The tapping mode elastic modulus measurement technique [24-29] provides another relatively new method for measuring the reduced elastic modulus. The measurement is based on the oscillation frequency of a probe that is in elastic contact with the surface. The tapping mode technique is nondestructive, which provides an advantage of measuring the elastic modulus prior to plastic deformation whereas nanoindentation measures the elastic-plastically deformed material.

The tapping mode method is now pursued for evaluating the Young's modulus of nanocrystalline metal coatings. Vapor deposited coatings of cubic metals can have a single-growth texture that is of interest for assessing the sensitivity of the measurement technique to anisotropic elastic behavior in nanocrystalline metals such as nickel. For this purpose, polycrystalline samples provide a basis for measurement calibration along with single-crystal wafer specimens. Further development of the classic Hertz-contact solution using higher-order terms of a Taylor's series expansion shows a quadratic rather than a linear relationship between the reduced elastic modulus and the resonant frequency shift. The new solution can be used to simulate the magnitude of the shift in the resonant frequency as a function of the reduced elastic modulus using the cantilever bending stiffness and the indenter tip radius of curvature.

\section{Materials and Methods}

2.1. Materials. The materials for the tapping mode measurement should have smooth surfaces. If there is a high amplitude to short-wavelength surface roughness, then multiple point contact can occur which could appear to artificially stiffen the mechanical response. Smooth surfaces and a nanocrystalline structure for tantalum $(\mathrm{Ta})$, vanadium $(\mathrm{V})$, and nickel $(\mathrm{Ni})$ coatings are produced from the high-quench rates $\left(>10^{6} \mathrm{~K} \cdot \mathrm{s}^{-1}\right)$ of the sputter deposition method [3034]. The metals are coated onto polished flats such as semiconductor-grade silicon and sapphire wafers. In brief, the $0.3-0.6 \mu \mathrm{m}$ thick coatings were synthesized by planarmagnetron sputter deposition in a vacuum chamber cryogenically pumped to a $2 \times 10^{-5} \mathrm{~Pa}$ base pressure. The native Si oxide on the substrate is not removed prior to deposition of the coating from target materials of $>0.999$ purity. The cathodes are operated in the DC mode with a typical discharge potential of $2 \times 10^{2}$ Volts. The source-to-substrate separation is $7-10 \mathrm{~cm}$ and the $1 \mathrm{~Pa}$ working-gas pressure of argon is maintained with a $35 \mathrm{~cm}^{3} \cdot \mathrm{s}^{-1}$ flow rate. The measured surface temperature of the substrate remains below $473 \mathrm{~K}$ during the deposition process. The deposition rates and thickness accumulation during deposition are recorded using calibrated $\mathrm{Au}$-coated quartz-crystal microbalances.

Details of the structural characterization for the Ta, V, and Ni sputter deposited coatings are reported elsewhere [30, 35]. The characterization methods consisted of X-ray diffraction (XRD), transmission electron microscopy (TEM), and atomic force microscopy (AFM). The XRD measurements were conducted in the $\theta / 2 \theta$ mode to determine both the crystalline structure and orientation. The sputter deposited metal coatings have a single growth orientation of close-packed planes. The body-center-cubic (bcc) metals of Ta and V are (110), and the face-center-cubic (fcc) $\mathrm{Ni}$ is (111). TEM imaging in bright field, in the electron-diffraction mode, and the use of high resolution lattice imaging conditions $[36,37]$ enable a closer look at the grain size, grain boundary structure, and defect structure. Selected area diffraction patterns (SADPs) confirm the (110) growth for the Ta and V coatings as well as the (111) growth for the Ni coating. Also, the SADPs indicate that the coatings are polycrystalline in plane, that is, randomly oriented parallel to the surface. The grain sizes determined from the average width to the columnar growth as seen in the bright field TEM images range from 14 to $20 \mathrm{~nm}$ for the $\mathrm{Ta}$ and $\mathrm{V}$ coatings to less than $150 \mathrm{~nm}$ for the Ni coating. AFM images [35] of the Ta and V surfaces indicate a smooth surface with less than a $1-2 \mathrm{~nm}$ variation in amplitude over $1 \mu \mathrm{m}$ length scales, that is, a surface smooth for establishing a Hertz-contact condition.

Semiconductor-grade, single-side polished wafers of silicon $\mathrm{Si}(100), \mathrm{Si}(111)$, and sapphire $\mathrm{Al}_{2} \mathrm{O}_{3}(00.2)$ are used to provide smooth single-crystal reference materials. Also, polished polycrystalline samples of polycarbonate $(\mathrm{pC})$, quartz $\left(\mathrm{SiO}_{2}\right)$, hydroxyapatite (HA), and sapphire $\left(\mathrm{Al}_{2} \mathrm{O}_{3}\right)$ are used as reference standards with known elastic constants for the establishment of the calibration curve from the tapping mode measurements.

2.2. Tapping Mode Experiment. The tapping mode use [24, $26,28]$ of a nanoprobe, in the configuration of a scanning force microscope, provides a method for measuring elastic deformation. A universal micro-nano-materials tester 
(UMNT), as manufactured by Bruker-Nano CETR, Inc., is equipped with a NanoAnalyzer (NA)-II test module that contains a ceramic cantilever with a Berkovich diamond tip. Both the $14 \mathrm{kHz}$ resonant frequency $\left(f_{0}\right)$ of the free-standing cantilever with a spring constant $\left(k_{c}\right)$ of $22.229 \mathrm{kN} \cdot \mathrm{m}^{-1}$ and the initial $5 \mathrm{~nm}$ amplitude $\left(A_{m}\right)$ of motion are changed as the probe is lowered so that contact is made with the specimen surface. The frequency $(f)$ and amplitude $\left(A_{m}\right)$ of the probe oscillation are quantitatively measured as a function of the vertical displacement $(x)$ of the probe tip. A frequency feedback system [29] moves the probe into the material surface until a predefined frequency shift $(\Delta f)$ is achieved that is typically less than $500-900 \mathrm{~Hz}$. In the elastic regime, the net elastic displacement $(z)$ beyond the initial surface contact is only a few nanometers, that is, $1-6 \mathrm{~nm}$, for most metals that accompany a decrease in the vibration amplitude from 5 to less than $0.2 \mathrm{~nm}$. A series of frequency shift curves, often a dozen or more, are used to achieve accuracy. The limited range of frequency shift $(\Delta f)$ available for elastic deformation from the free-standing resonant frequency $\left(f_{0}\right)$ in the NA-II precludes access to an examination of higherorder harmonics.

The $\Delta f$ versus $z$ variation of the tip-surface system can be equated as a function of the reduced elastic modulus $\left(E^{*}\right)$. The dynamic behavior of the indenter probe in contact with the surface is derived from the equation of motion and the equation for the frequency of oscillation of the system. A formulation of the $\alpha$-parameter is determined [24,25] from a Taylor series expansion of the equations to the Hertz-contact model for the variation of $\Delta f$ with $z$ as

$$
\alpha^{2}=\frac{(\Delta f)^{2}}{z} .
$$

The relationship between the square of the resonant frequency shift $(\Delta f)^{2}$ and the vertical displacement $(x)$ of the cantilever is seen in the schematic in Figure 1 that has four distinct regions where (1) the tip oscillates without surface contact, (2) there is contact between the tip and a viscous (contamination) surface layer, (3) direct interaction of the tip occurs with atoms of the specimen surface, and (4) damping of the probe oscillation is seen due to plastic deformation. Region 3 has two portions. The amplitude of the tip oscillation is still large and the probe base is far from the surface in region $3^{\prime}$; that is, the tip is bouncing off the surface. As $x$ increases, the tip and surface begin to oscillate in region $3^{\prime \prime}$ without separation in a strict contact mode, that is, the elastic regime corresponding with (3). In almost all cases, the amplitude of the oscillation can have a significant role in determining the extent of the linear regime in region $3^{\prime \prime}$. The length of the linear elastic regime is reduced when the amplitude of oscillation is large with respect to the tip radius. In general, the amplitude-to-tip radius ratio is in the order of $5 \%$ or less. An initial vibration amplitude of $5 \mathrm{~nm}$ works well for a wide range of materials using a Berkovich-shaped diamond tip. The material starts to plastically deform in region 4 of Figure 1 as the probe is pressed further into the surface where the associated deformation imparts a damping action on the vibration of the probe. The result is a sudden change in the

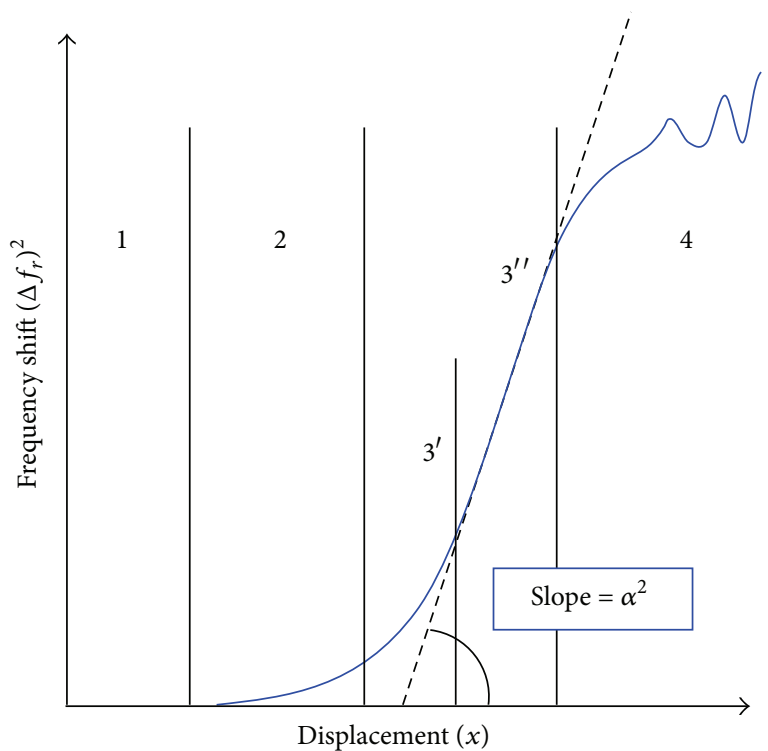

FIGURE 1: A plot of the characteristic regions observed for variation in the square of the resonant frequency shift $\left(\Delta f_{r}\right)^{2}$ with displacement $(x)$ when the probe tip mounted to a vibrating cantilever comes in contact with a surface.

frequency shift from the linear regime of region $3^{\prime \prime}$ as the amplitude fully diminishes. This phenomenon is observed for a wide range of materials such as metals and ceramics. The series of frequency shift curves are aligned where the amplitude reduces to zero to define the upper limit to region $3^{\prime \prime}$. As a visual confirmation, the displacement $x$ for the region $3^{\prime \prime}$ upper limit position should not proceed beyond the square of the frequency shift where an indentation is observed on the imaged surface, that is, plastic deformation. To determine the lower limit to region $3^{\prime \prime}$ that bounds the onset of the elastic response, care must be taken to avoid the preceding nonlinear section of the curve as shown by the vertical lines that bound region $3^{\prime \prime}$ in Figure 1. Once the linear regime is defined, as, for example, by a correlation coefficient $R_{c c^{2}}>0.98$, the slope of region $3^{\prime \prime}$ is determined from (3).

The calibrated measurement of the reduced elastic modulus $\left(E^{*}\right)$ for an unknown material is accomplished from tapping mode measurements of $\alpha^{2}$ using the following generalized expression:

$$
\alpha=a\left(E^{*}\right)^{n} .
$$

In (4), use is made of a power-law exponent $(n)$ and a scaling constant $(a)$. This generalized expression can accommodate the most basic form of a nonlinear relationship between $E^{*}$ and $\alpha$, as is commonly found in experimental measurements. A two-term Taylor series expansion [24, 25] for the solution of the Hertz-contact condition between tip and surface yields an exponent $n$ value of one, that is, a linear relationship. In the next section, the more satisfactory formulation of a generalized nonlinear relationship between $E^{*}$ and $\alpha$ will be seen to require additional terms in the Taylor series expansion. For the calibration test method, several unique measurements of $\alpha^{2}$ must be obtained for a variety of 
known material standards from which a calibration curve is plotted using (4). In this way, the basis is established for an experimental calibration curve that corresponds with the particular cantilever-tip configuration. The calibration curve is made using materials with well-established elastic moduli $(E)$ to cover a large $E^{*}$ range from 3 to $400 \mathrm{GPa}$. The determination of $E^{*}$ for the unknown material proceeds with (4) using an input of the measured $\alpha$ value for that material. The NanoAnalyzer NA-II is used as well to scan the sample surfaces to avoid defected regions and to confirm the smoothness required for the tapping mode measurements.

\section{Results and Discussion}

3.1. Tapping Mode Analytic Model. An analytic method [38] is used to determine Young's modulus $E$ through derivation of exact expressions for $\alpha$ and $E^{*}$ as well as the final form of the elasticity equation from Hertz-contact mechanics. The formulations presented in (3) and (4) are justified using the dynamic equivalent of the cantilever with a probe tip. This dynamic system can be represented [26] by a spring-mass system where the equation of motion is given as

$$
m \ddot{x}+k_{c} x=0 \text {. }
$$

In (5), the mass is $m$, the displacement is $x$, and the spring constant of the cantilever $k_{c}$ is given by

$$
k_{c}=\frac{3 E I}{L^{3}}
$$

In (6), the cantilever's moment of inertia is $I$, the elastic modulus is $E$, and its length is $L$. Thus, the natural oscillation frequency of the system is described as

$$
\omega_{n}=\sqrt{\frac{k_{c}}{m}}=2 \pi f_{0} .
$$

When the probe is in contact with the surface, the dynamic system is modeled such that, for a displacement $x$, the frequency of oscillation of the system $f$ is given as

$$
2 \pi f=\sqrt{\frac{k_{c}+k_{s}}{m}} .
$$

In (8), $k_{s}$ is the spring constant to the elastic response of the surface. Combining (7) and (8) and solving for $f$ yield the expression

$$
f=f_{0}\left(\sqrt{1+\frac{k_{s}}{k_{c}}}\right) .
$$

The change of frequency from the state of natural oscillation to the contact state with the surface is then given by

$$
\Delta f=f-f_{0}=f_{0}\left(\sqrt{1+\frac{k_{s}}{k_{c}}}-1\right) .
$$

A Taylor series expansion of (9) using only the first two terms, combined with (10), gives the previously well-known simplified expression for $\Delta f$ as

$$
\Delta f=f_{0}\left(\frac{k_{s}}{2 k_{c}}\right) .
$$

From Hertz-contact mechanics, it is known that, after application of normal load $(P)$, the distance $(z)$ from the plane of the surface contact is given by

$$
z=\left(\frac{3 P}{4 E^{*}}\right)^{2 / 3}\left(\frac{1}{R}\right)^{1 / 3} .
$$

In (12), the hemispherical tip radius is $R$. The spring constant for the surface $\left(k_{s}\right)$ can be modeled using the relationship

$$
k_{s}=\frac{\partial P}{\partial z}
$$

After inserting (12) into the operation for (13), an expression for $k_{s}$ then results as

$$
k_{s}=\frac{\partial P}{\partial z}=2 \sqrt{R} E^{*} \sqrt{z} .
$$

Using (14) expression for $k_{s}$ and inserting it into (11) yield an expression for the frequency shift $(\Delta f)$ as

$$
\Delta f=\frac{f_{0} \sqrt{R}}{k_{c}} E^{*} \sqrt{z} .
$$

After taking a square of both sides to (15), a relationship is now derived between $(\Delta f)^{2}$ and the displacement $(z)$ as

$$
(\Delta f)^{2}=\alpha^{2} z
$$

where

$$
\alpha=\frac{f_{0} \sqrt{R}}{k_{c}} E^{*} .
$$

A plot of the square of frequency shift $(\Delta f)^{2}$ versus the probe tip-surface elastic displacement $(z)$, as first presented in the similar expression of (4), is used to experimentally measure the slope $\alpha^{2}$ that corresponds with (16). The expression (17) that shows a linear relationship between $E^{*}$ and $\alpha$, as derived elsewhere $[25,26]$, uses only the first two terms of the Taylor's series expansion for (10). Therefore, in order to determine a more exact analytic expression for the relationship between $\alpha$ and $E^{*}$ that accommodates a nonlinear relationship as expressed in the generalized power-law expression of (4), a third term is now introduced into the Taylor's series expansion. This procedure provides an increase in accuracy in comparison to (11) for determining the relationship between the frequency shift and elastic modulus using (14). The series expansion used to derive (11) and (17) for $\alpha$ now becomes the following expression that is derived from (9) and (10):

$$
\alpha=\frac{f_{0} \sqrt{R}}{k_{c}} E^{*}\left[1-\frac{1}{2}\left(\sqrt{R} E^{*}\right) \frac{\sqrt{z}}{k_{c}}\right] .
$$


TABle 1: Values for $\alpha$, displacement $(z)$, Poisson's ratio $(\nu)$, and elastic modulus $E^{*} ; E(h k l)$.

\begin{tabular}{|c|c|c|c|c|c|c|c|c|}
\hline \multirow{2}{*}{ Sample } & \multirow{2}{*}{$\alpha\left(\mathrm{Hz} \cdot \mathrm{nm}^{-1 / 2}\right)$} & \multirow{2}{*}{$z(\mathrm{~nm})$} & \multirow{2}{*}{$E^{*}(\mathrm{GPa})$} & \multirow{2}{*}{$v$} & \multirow{2}{*}{$E(\mathrm{GPa})$} & \multicolumn{3}{|c|}{ Cubic materials $E(h k l)(\mathrm{GPa})$} \\
\hline & & & & & & (111) & (110) & $(100)$ \\
\hline Polycarbonate $\mathrm{pC}$ & $35.8 \pm 1.4$ & 5.08 & 4 & 0.37 & 3.5 & - & - & - \\
\hline Fused silica $\mathrm{SiO}_{2}$ & $145 \pm 14$ & 4.17 & 70 & 0.17 & 72 & - & - & - \\
\hline Fused quartz $\mathrm{SiO}_{2}$ & $149 \pm 13$ & 3.25 & 70 & 0.17 & 72 & - & - & - \\
\hline Hydroxyapatite HA & $170 \pm 15$ & 1.46 & 86 & 0.27 & 85 & - & - & - \\
\hline Silicon $\operatorname{Si}(100)$ & $202 \pm 16$ & 1.18 & 127 & 0.27 & 130 & 188 & 169 & 130 \\
\hline Vanadium V(110) & $228 \pm 22$ & 1.63 & 130 & 0.37 & 125 & 119 & 125 & 147 \\
\hline Silicon Si(111) & $236 \pm 23$ & 2.74 & 176 & 0.27 & 188 & 188 & 169 & 130 \\
\hline Tantalum $\mathrm{Ta}(110)$ & $271 \pm 23$ & 1.62 & 187 & 0.34 & 192 & 216 & 192 & 144 \\
\hline Nickel Ni(111) & $325 \pm 20$ & 1.71 & 269 & 0.31 & 305 & 305 & 227 & 129 \\
\hline Fused sapphire $\mathrm{Al}_{2} \mathrm{O}_{3}$ & $401 \pm 26$ & 0.98 & 367 & 0.27 & 470 & - & - & - \\
\hline Sapphire $\mathrm{Al}_{2} \mathrm{O}_{3}(00.2)$ & $392 \pm 27$ & 0.78 & 382 & 0.27 & 496 & - & - & - \\
\hline
\end{tabular}

Equation (18) can be reduced to the form of (17). Formula (18) can be solved for $E^{*}$ as a function of $f_{0}, \alpha, z, k_{c}$, and $R$ by using a standard quadratic expression for $E^{*}$ as

$$
E^{*}=\frac{1 \pm \sqrt{1-\left(2 \alpha \sqrt{z} / f_{0}\right)}}{\sqrt{R z} / k_{c}} .
$$

Experimentally meaningful values are produced from (19) using the $(1-\sqrt{ })$ term in the numerator. Equations (3) and (4) become the basis for the calibration procedure to curve-fit the coefficient $(a)$ and exponent $(n)$ values to the power-law relationship and to be used for the determination of unknown $E^{*}$ values. The nonlinear algebraic solution of (18) indicates a quadratic relationship between $\alpha$ and $E^{*}$ (as dependent on $\sqrt{R}$ and $\sqrt{z}$ ) to reveal that the corresponding value of the exponent $n$ for the power-law fit to (4) may be approximately $1 / 2$.

The reduced elastic modulus $\left(E^{*}\right)$ of the sample can be computed from (19) provided that measurements are possible for the free-standing oscillation frequency $f_{0}$ of the cantilever, the cantilever bending stiffness $k_{c}$, the probe tipsurface displacement $z$, and probe tip radius $R$. The calibration method for measurement of reduced elastic modulus $E^{*}$ is more frequently used since the probe tip values are often determined with limited accuracy. In the calibration method, the $\alpha$ values are measured as described previously using a particular probe tip for several materials with known elastic modulus. The $\alpha$ values are then plotted as a function of the corresponding known reduced modulus values in accordance with (4) to fit the parameters for the coefficient $a$ and power-law exponent $n$. The greater the number of calibration materials is, the better the power-law curve fit will be for a more accurate calculation of unknown elastic moduli. Use of (19) will be made to simulate (4) curve fit results and to determine an approximate value for the indenter tip radius $R$ as may be dependent upon $z$.

3.2. Computation of Elastic Moduli. The effect of crystalline anisotropy on the elastic moduli can be quantified using a mathematical formulation [39] of the elastic moduli $(E)$ and tabulated values [40-48] for the elastic constants $\left(C_{i j}\right)$ and compliances $\left(S_{i j}\right)$. The equation for Young's modulus $E(h k l)$ for cubic systems along the $\langle h k l\rangle$ directions with respect to the crystalline orientation using the unit vectors $\left(l_{i}\right)$ such as those designated by the Miller indices of $\langle 100\rangle,\langle 110\rangle$, and $<111>$ is

$$
\frac{1}{E}=S_{11}-2\left(S_{11}-S_{12}-\frac{1}{2} S_{44}\right)\left(l_{1}^{2} l_{2}^{2}+l_{2}^{2} l_{3}^{2}+l_{3}^{2} l_{1}^{2}\right) .
$$

For the crystal systems with $3 \mathrm{~m}, 32$, and $-3 \mathrm{~m}$ classes of symmetry, the expression for Young's modulus becomes

$$
\begin{aligned}
\frac{1}{E}= & \left(1-l_{3}^{2}\right)^{2} S_{11}+l_{3}^{4} S_{33} \\
& +l_{3}^{2}\left(1-l_{3}^{2}\right)\left(2 S_{13}+S_{44}\right) \\
& +2 l_{1}^{2} l_{3}^{2}\left(3 l_{1}^{2}-l_{2}^{2}\right) S_{14} .
\end{aligned}
$$

The $E(h k l)$ values computed using (20) and (21) are listed in Table 1 for the comparison of anisotropy within each and all of the materials in the order of increasing stiffness. The listed $E^{*}$ values are computed using $(2)$ assuming a $\left(1-v_{t}^{2}\right) / E_{t}$ value of $0.00075 \mathrm{GPa}^{-1}$ for the probe tip as based upon a nanoindentation calibration of the Berkovich diamond tip to the known hardness value for silicon. The $\left(1-v_{t}^{2}\right) / E_{t}$ value of $0.00075 \mathrm{GPa}^{-1}$ is consistent with the typical $\nu_{t}$ values of $0.07-$ 0.10 and $E_{t}$ values of $1150-1250 \mathrm{GPa}$ that are often reported for diamond.

3.3. Tapping Mode Measurements. The square of the resonant frequency shift $\left(\Delta f_{r}\right)^{2}$ with probe displacement $x$ for the nanocrystalline $\mathrm{V}(110)$ and $\mathrm{Ni}(111)$ coatings is shown in the plots of Figures 2(a) and 3(a). For the V(110) example, an average value of $85 \times 10^{4} \mathrm{~Hz}^{2}$ is computed for the change in $\left(\Delta f_{r}\right)^{2}$ that corresponds to the $1.63 \mathrm{~nm}$ portion of the linear elastic displacement $(z)$ from region $3^{\prime \prime}$ in Figure 2(a), centered at a displacement $(x)$ of $28.7 \mathrm{~nm}$. The data plots of Figures 2(b) and 3(b) show the simultaneous variation of the oscillation amplitude $A_{m}$ with the probe tip displacement $(x)$. 


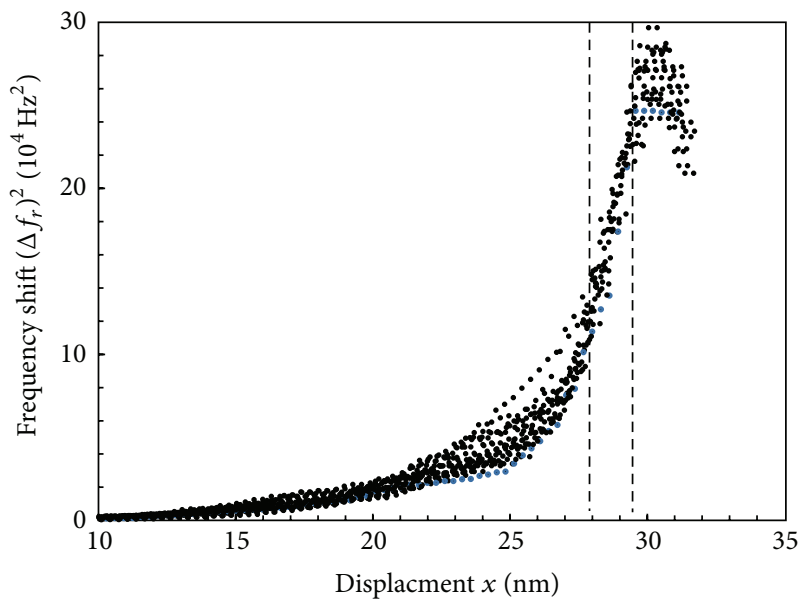

(a)

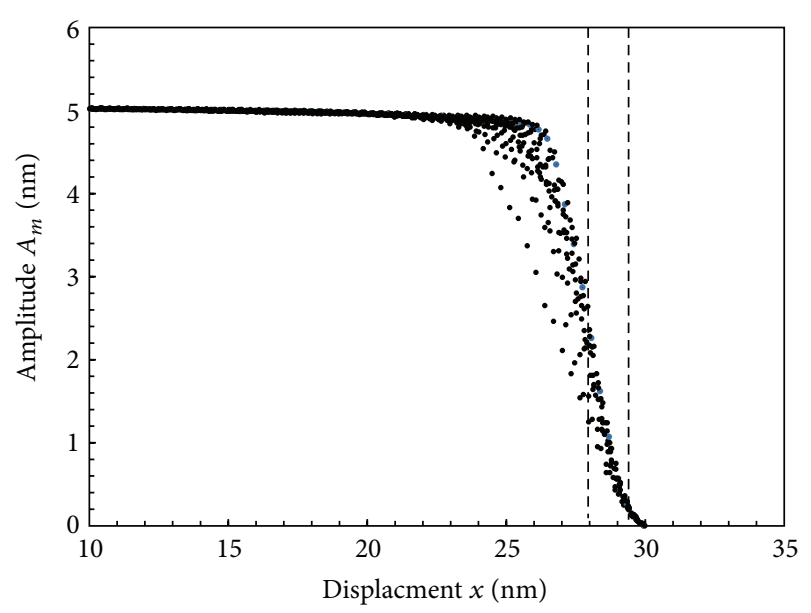

(b)

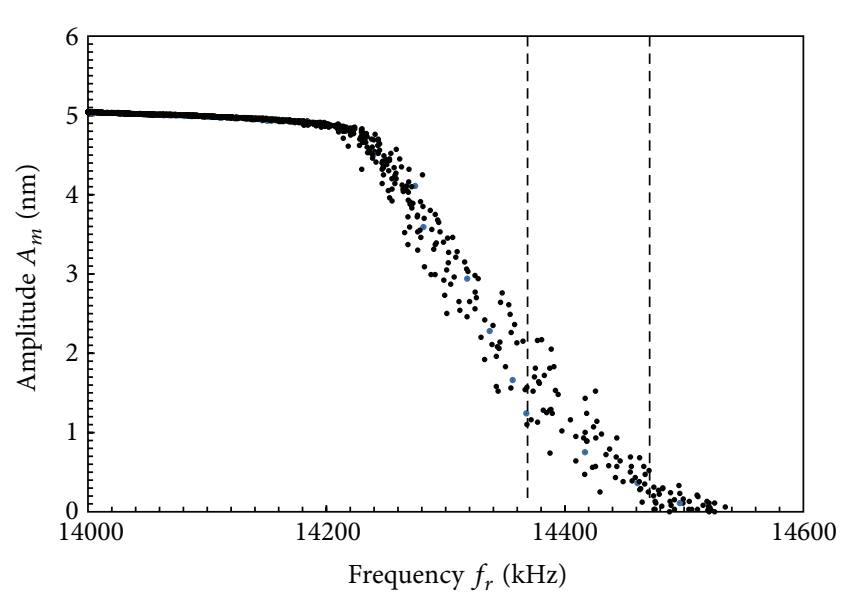

(c)

FIgURE 2: (a) The square of the resonant frequency shift $\left(\Delta f_{r}\right)^{2}$ variation with the probe tip displacement $x$ and the amplitude $A_{m}$ of the oscillation variation with (b) the probe tip displacement $x$ and (c) resonant frequency $f_{r}$ are shown for a nanocrystalline $\mathrm{V}(110)$ coating deposited onto a Si substrate.

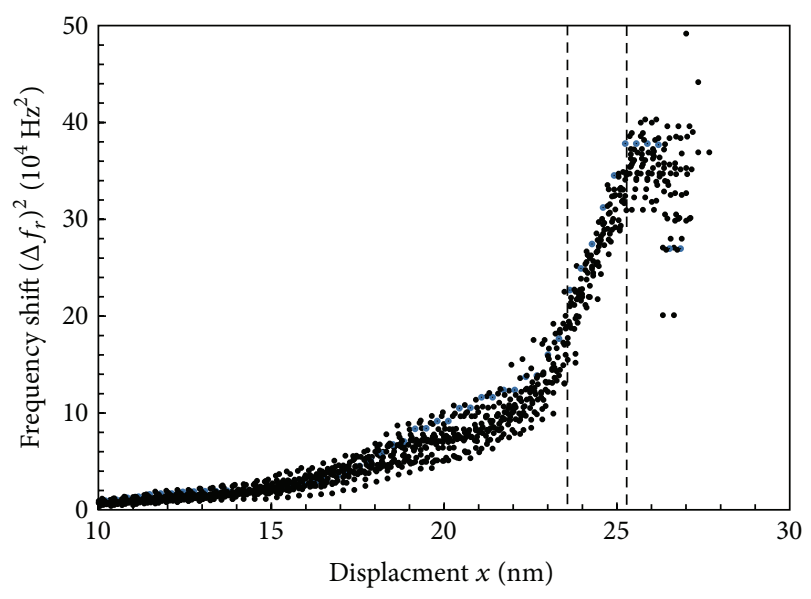

(a)

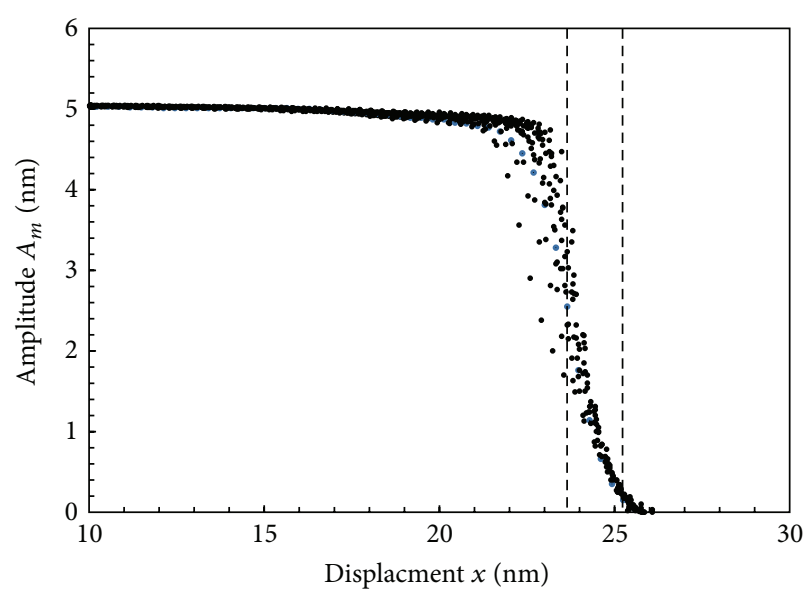

(b)

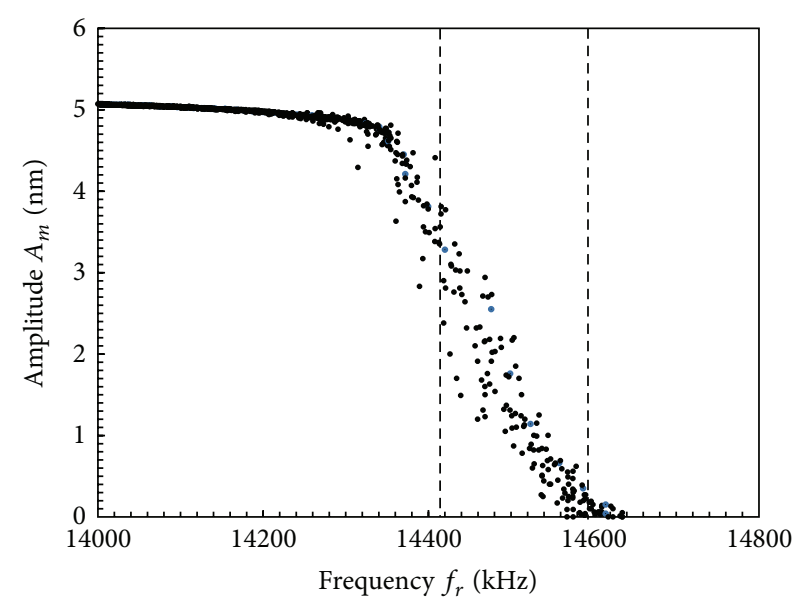

(c)

FIgURE 3: (a) The square of the resonant frequency shift $\left(\Delta f_{r}\right)^{2}$ variation with the probe tip displacement $x$ and the amplitude $A_{m}$ of the oscillation variation with (b) the probe tip displacement $x$ and (c) resonant frequency $f_{r}$ are shown for a nanocrystalline $\mathrm{Ni}(111)$ coating deposited onto a Si substrate. 


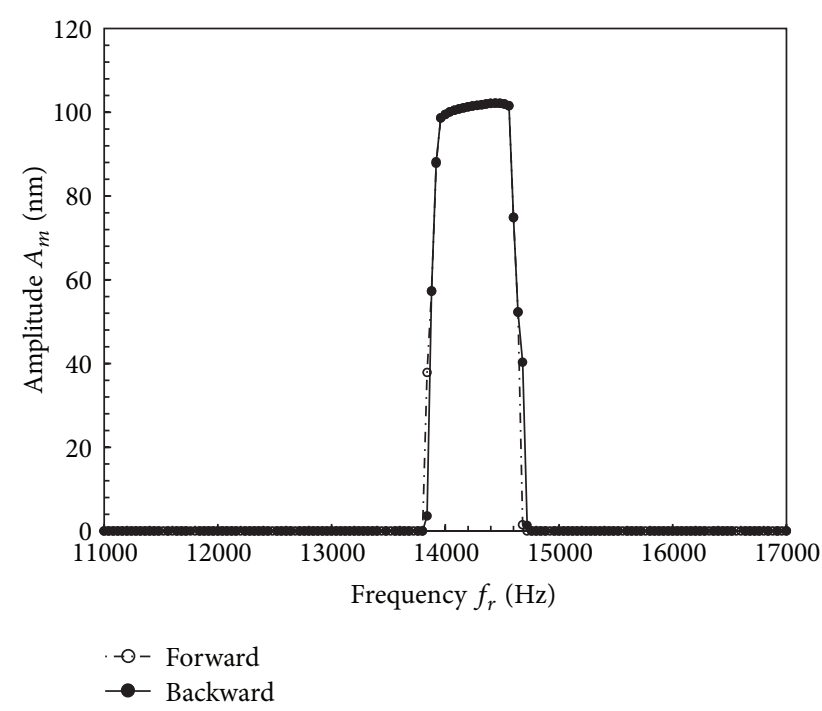

Figure 4: The amplitude $A_{m}$ of the oscillation variation with the resonant frequency $\left(f_{r}\right)$ for the free-standing cantilever.

The magnitude of $A_{m}$ decreases to only $0.2 \mathrm{~nm}$ in region $3^{\prime \prime}$ from its original region 1 value of $5 \mathrm{~nm}$ when free standing.

The decrease in the amplitude $A_{m}$ for the $\mathrm{V}(110)$ and $\operatorname{Ni}(111)$ samples with increasing resonant frequency $\left(f_{r}\right)$ is shown in Figures 2(c) and 3(c). The vertical markers correspond with the changes in the square of the resonant frequency shift indicated in Figures 2(a) and 3(a) for the $\mathrm{V}(110)$ and $\mathrm{Ni}(111)$ samples. For comparison, a forward and backward scan through the resonant frequency of the freestanding cantilever is shown in Figure 4.

The results for the calculations of the $\alpha$ values for all of the samples that are determined from the tapping mode measurements of the change in $\left(\Delta f_{r}\right)^{2}$, according to (3), are listed in Table 1. The results for the reduced elastic modulus $E^{*}(\mathrm{GPa})$ calibration curve as plotted with the measured $\alpha\left(\mathrm{Hz} \cdot \mathrm{nm}^{-1 / 2}\right)$ values are shown in Figure 5 by the solid symbols. The power-law curve fit to (4) gives a value for $n$ equal to 0.532 and value for $a$ equal to 16.2 $\left(\mathrm{Hz} \cdot \mathrm{nm}^{-1 / 2} \cdot \mathrm{GPa}^{-n}\right)$, where the correlation coefficient $R_{c c^{2}}$ to the curve fit equals 0.994 .

The computation of the Table 1 values for $E^{*}$ from the known $E$ values is made using (2). The individual (opensymbol) data points for the elastic moduli of non-closepacked growth planes are plotted to evaluate the effect of crystalline orientation on the calibration curve fitting of the measured elastic modulus for the cubic nanocrystalline metal coatings. The values that are computed for $\mathrm{Ni}(100), \mathrm{Ni}(110)$, $\mathrm{V}(100), \mathrm{V}(111), \mathrm{Ta}(100)$, and $\mathrm{Ta}(111)$ using (20)-(21) are listed in Table 1. Clearly, the modulus values for $\mathrm{Ni}(100), \mathrm{Ni}(110)$, and $\mathrm{Ta}(100)$ cannot be considered as these values rest well off (4) calibration curve. In addition, a poor curve fit to (4) results using averaged $E(h k l)$ values for Ta, $\mathrm{Ni}$, and $\mathrm{V}$. The modulus that corresponds to the single growth orientation of the nanocrystalline metal coating is consistent with (4) curve fit of modulus values for the polycrystalline and singlecrystal standards. Therefore, in general, it appears that the

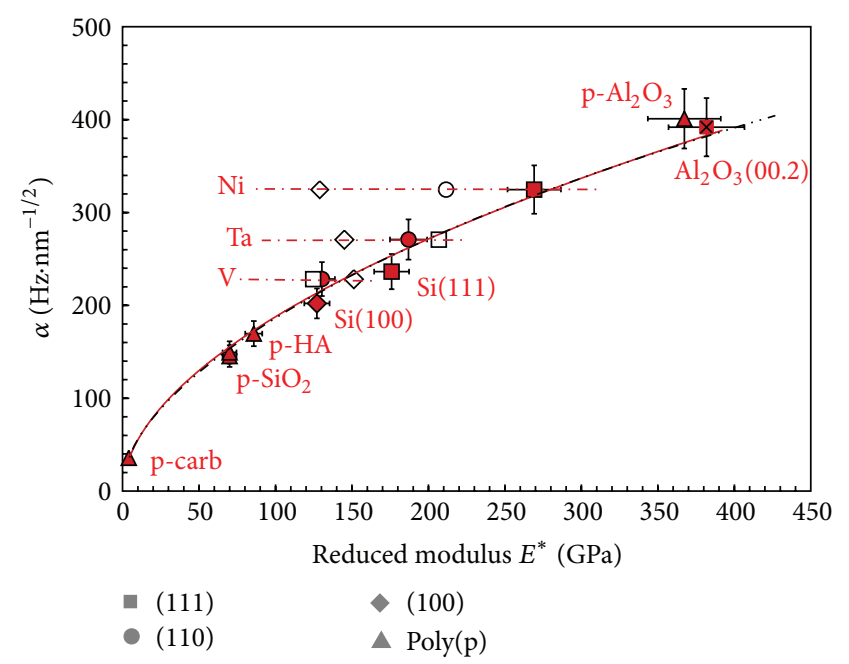

FIGURE 5: The measured $\alpha$ value ( $s$ ) that corresponds with the square root of the slope in region $3^{\prime \prime}$ (of Figure 1) is plotted as function of the reduced modulus $E^{*}$ value for various sample materials where the crystalline orientation is indicated by the symbol shape in the legend.

measured Young's modulus values correspond to the actual growth orientation of the nanocrystalline metal coatings.

These present results for the variation of $E^{*}$ with $\alpha$ are consistent with the polycrystalline calibration materials and the elastic anisotropy of the single-crystal samples of $\mathrm{Si}(100)$, $\mathrm{Si}(111)$, and $\mathrm{Al}_{2} \mathrm{O}_{3}(00.2)$, as well as the elastically anisotropic nanocrystalline $\mathrm{Ta}(110), \mathrm{V}(110)$, and $\mathrm{Ni}(111)$ coatings. For comparison, similar elastic modulus values of $187 \mathrm{GPa}$ and $141 \mathrm{GPa}$ are reported [35] for the $\mathrm{Ta}(110)$ and $\mathrm{V}(110)$ coatings, respectively, as determined from nanoindentation tests. However, the nanoindentation values do appear to include some contribution of a (100) component during elastic unloading of the coating subsequent to plastic deformation.

The $E^{*}$ values that correspond with the actual crystalline orientation of the coatings are used in the (solid-line) curve fit to (4). A (dashed-line) curve that represents the analytic model approach is computed using (19). The analytic model curve overlays the calibration curve fit. The analytic model (dashed-line) curve in (19) is formed by computing the $E^{*}$ and $\alpha$ values using tip radius values $(R)$ that are fitted to the corresponding elastic displacement condition for each sample to reproduce the experimental (solid-line) calibration curve. The tip radius $(R)$ variation with elastic displacement is reproduced as generally anticipated for diamond indenters. An $R$ value of $10^{2} \mathrm{~nm}$ is modeled at the larger depths of full elastic displacement $(z)$. (Only a portion of the elastic displacement $z$ used in the $\alpha$ value computations is listed in Table 1.) In general, the elastic displacement progressively decreases as the samples stiffen. Whereas a very sharp tip radius and nanometer-scale elastic deformation could clearly present the need to consider modeling of near-atomic interactions, the use of a classic modeling approach as presented for Hertzian tests is generally consistent with the actual diamond tip condition where the contact radius is less than $5 \%$ of the tip radius. The utility of the classical model is 
supported by the self-consistent experimental measurements of the calibration materials with known elastic behavior.

3.4. Discussion. The sensitivity to potential elastic anisotropy is seen in the measurement of the Young's modulus values that correspond with surface normal loading of the tapping mode method. The elastic response is measured in the direction of growth for the nanocrystalline coatings, as well as the single-crystal wafer orientation seen in the different $\alpha$ values measured for $\mathrm{Si}(100)$ and $\mathrm{Si}(111)$. The present result indicates that measurement of Young's modulus is directionally dependent on loading. Modulus values determined with in-plane loading condition are not equivalent to the normal-incident loading by methods such as nanoindentation and the tapping mode.

The tapping mode measurement of thin film coatings using the resonant frequency shift is useful for a wide range of materials with smooth surfaces. The method is easily applied when the indenter tip is much stiffer than the surface being measured. The use of higher-order terms in the Taylor series expansion of the linear solution for $\alpha$ in (17) results in the more general, nonlinear solution as presented in (18). This extension can be advantageous in modeling experimental data since it is difficult to meet the assumption of the first-order approximation that $k_{s} \ll k_{c}$ for typical cantilevers, where $k_{c} \sim 10^{4} \mathrm{kN} \cdot \mathrm{m}^{-1}$ and $R \sim$ $10^{2} \mathrm{~nm}$. However, the analytic approach presented does not account for adverse effects of tip deformation, a pull-off force, or surface adhesion. Whereas the Hertz expression of (12) can hold true for large loads [49, 50], an overestimation of the elastic modulus at low loads can occur when the effect of surface energy $(\gamma)$ is significant. Here, the JohnsonKendall-Roberts (JKR) model can provide a better estimation of the contact radius. Similarly, an accurate expression can be derived [51] from the Derjaguin-Muller-Toporov (DMT) model that involves a Taylor series expansion with more than just the first two terms. The amount of pull-off force can be determined by conducting future nanoindentation experiments [52, 53]. The measurement of pull-off force requires careful chemical cleaning of the sample surfaces. At present, the tapping mode measurements are made with the surface-contamination layer intact which contains hydrocarbons and appears to provide a natural lubrication alleviating background adhesion effects.

\section{Conclusions}

Young's modulus $E$ is measured using the tapping mode of a cantilever-mounted diamond probe. The shift in the resonant frequency $f_{0}$ of the cantilever is recorded when the diamond probe is in elastic contact with the sample surface. The variation in the square of the change in the resonant frequency $\left(\Delta f_{r}\right)^{2}$ divided by the accompanying elastic displacement $z$ is equated to an experimental $\alpha^{2}$ parameter; that is, $\alpha^{2}$ equals $\left(\Delta f_{r}\right)^{2} / z$. A power-law relationship is used to equate $\alpha$ with the reduced elastic modulus $E^{*}$. An analytic derivation, using higher-order terms from a Taylor series expansion of the solution to the Hertz condition for the probe and surface, indicates that the exponent to the power-law relationship approximates a quadratic expression between $\alpha\left(\mathrm{Hz} \cdot \mathrm{nm}^{-1 / 2}\right)$ and $E^{*}(\mathrm{GPa})$. For the Berkovich tip of this study, $\alpha$ equals $16.2 \cdot\left(E^{*}\right)^{0.532}$.

Tapping mode measurements are made of sputter deposited tantalum $(\mathrm{Ta})$, vanadium $(\mathrm{V})$, and nickel $(\mathrm{Ni})$ coatings that have a close-packed plane and growth orientation. Polycrystalline ceramics and single-crystal wafers of $\mathrm{Si}(100)$, $\mathrm{Si}(111)$, and $\mathrm{Al}_{2} \mathrm{O}_{3}(00.2)$ are used to establish the $\alpha$ versus $E^{*}$ calibration curve along with measurements of the nanocrystalline metal coatings. The tapping mode results indicate that Young's modulus measurement corresponds with the crystalline orientation that is normal to the sample surface, and in the loading direction of the elastic displacement. For example, a measured $\alpha$ value of $325 \mathrm{~Hz} \cdot \mathrm{nm}^{-1 / 2}$ corresponds with a $305 \mathrm{GPa}$ Young's modulus for a nanocrystalline $\mathrm{Ni}(111)$ coating. The directional behavior reveals sensitivity to elastic anisotropy for a variety of known materials over a 3-400 GPa range of elastic modulus using a diamond tip probe.

\section{Acknowledgments}

This work was supported by the J.W. Wright and Regents Endowments for Mechanical Engineering at Texas Tech University (TTU). The authors thank Dr. Ilja Hermann for reviewing the paper and for his instructive comments.

\section{References}

[1] T. G. Nieh and J. Wadsworth, "Hall-petch relation in nanocrystalline solids," Scripta Metallurgica et Materiala, vol. 25, no. 4, pp. 955-958, 1991.

[2] C. A. Schuh, T. G. Nieh, and H. Iwasaki, "The effect of solid solution $\mathrm{W}$ additions on the mechanical properties of nanocrystalline Ni," Acta Materialia, vol. 51, no. 2, pp. 431-443, 2003.

[3] A. F. Jankowski, C. K. Saw, J. F. Harper, B. F. Vallier, J. L. Ferreira, and J. P. Hayes, "Nanocrystalline growth and grain-size effects in Au-Cu electrodeposits," Thin Solid Films, vol. 494, no. 1-2, pp. 268-273, 2006.

[4] M. Dao, L. Lu, R. J. Asaro, J. T. M. De Hosson, and E. Ma, "Toward a quantitative understanding of mechanical behavior of nanocrystalline metals," Acta Materialia, vol. 55, no. 12, pp. 4041-4065, 2007.

[5] N. Tayebi, T. F. Conry, and A. A. Polycarpou, "Determination of hardness from nanoscratch experiments: corrections for interfacial shear stress and elastic recovery," Journal of Materials Research, vol. 18, no. 9, pp. 2150-2162, 2003.

[6] C. A. Schuh, T. G. Nieh, and T. Yamasaki, "Hall-Petch breakdown manifested in abrasive wear resistance of nanocrystalline nickel," Scripta Materialia, vol. 46, no. 10, pp. 735-740, 2002.

[7] K. M. Lee, C.-D. Yeo, and A. A. Polycarpou, "Nanomechanical property and nanowear measurements for Sub-10-nm thick films in magnetic storage," Experimental Mechanics, vol. 47, no. 1, pp. 107-121, 2007.

[8] J. Chen, L. Lu, and K. Lu, "Hardness and strain rate sensitivity of nanocrystalline Cu," Scripta Materialia, vol. 54, no. 11, pp. 19131918, 2006.

[9] L. O. Nyakiti and A. F. Jankowski, "Characterization of strainrate sensitivity and grain boundary structure in nanocrystalline 
gold-copper alloys," Metallurgical and Materials Transactions A, vol. 41, no. 4, pp. 838-847, 2010.

[10] M. F. Doerner and W. D. Nix, "A method for interpreting the data from a depth sensing indentation instrument," Journal of Materials Research, vol. 1, no. 4, pp. 601-609, 1986.

[11] W. C. Oliver and G. M. Pharr, "Improved technique for determining hardness and elastic modulus using load and displacement sensing indentation experiments," Journal of Materials Research, vol. 7, no. 6, pp. 1564-1583, 1992.

[12] W. C. Oliver and G. M. Pharr, "Measurement of hardness and elastic modulus by instrumented indentation: advances in understanding and refinements to methodology," Journal of Materials Research, vol. 19, no. 1, pp. 3-20, 2004.

[13] E. Z. Meyer, "Untersuchungen über Härteprüfung und Härte," Zeitschrift des Vereines Deutscher Ingenieure, vol. 52, pp. 645654, 1908.

[14] A. F. Jankowski, "Superhardness effect in Au/Ni multilayers," Journal of Magnetism and Magnetic Materials, vol. 126, no. 1-3, pp. 185-191, 1993.

[15] J. J. Vlassak and W. D. Nix, "Measuring the elastic properties of anisotropic materials by means of indentation experiments," Journal of the Mechanics and Physics of Solids, vol. 42, no. 8, pp. 1223-1245, 1994.

[16] J. G. Swadener and G. M. Pharr, "Indentation of elastically anisotropic half-spaces by cones and parabolae of revolution," Philosophical Magazine A, vol. 81, no. 2, pp. 447-466, 2001.

[17] A. Delafargue and F.-J. Ulm, "Explicit approximations of the indentation modulus of elastically orthotropic solids for conical indenters," International Journal of Solids and Structures, vol. 41, no. 26, pp. 7351-7360, 2004.

[18] F. A. McClintock and A. S. Argon, "Other measures of plastic hardness," in Mechanical Behavior of Materials, vol. 13 of Addison-Wesley Series in Metallurgy and Materials, pp. 450-458, Addison-Wesley, Reading, 1966.

[19] D. Tabor, “The hardness of solids," Review of Physics in Technology, vol. 1, no. 3, pp. 145-179, 1970.

[20] M. Reinstädtler, T. Kasai, U. Rabe, B. Bhushan, and W. Arnold, "Imaging and measurement of elasticity and friction using the TRmode," Journal of Physics D, vol. 38, no. 18, pp. R269-R282, 2005.

[21] D. DeVecchio and B. Bhushan, "Localized surface elasticity measurements using an atomic force microscope," Review of Scientific Instruments, vol. 68, no. 12, pp. 4498-4505, 1997.

[22] R. Whiting and M. A. Angadi, "Young's modulus of thin films using a simplified vibrating reed method," Measurement Science and Technology, vol. 1, no. 7, article 024, pp. 662-664, 1990.

[23] K.-D. Wantke, H. Fruhner, J. Fang, and K. Lunkenheimer, "Measurements of the surface elasticity in medium frequency range using the oscillating bubble method," Journal of Colloid and Interface Science, vol. 208, no. 1, pp. 34-48, 1998.

[24] A. S. Useinov, "A nanoindentation method for measuring the Young modulus of superhard materials using a NanoScan scanning probe microscope," Instruments and Experimental Techniques, vol. 47, no. 1, pp. 119-123, 2004.

[25] K. V. Gogolinskiǐ, Z. Y. Kosakovskaya, A. S. Useinov, and I. A. Chaban, "Measurement of the elastic moduli of dense layers of oriented carbon nanotubes by a scanning force microscope," Acoustical Physics, vol. 50, no. 6, pp. 664-669, 2004.

[26] S. I. Lee, S. W. Howell, A. Raman, and R. Reifenberger, "Nonlinear dynamics of microcantilevers in tapping mode atomic force microscopy: a comparison between theory and experiment,"
Physical Review B, vol. 66, no. 11, Article ID 115409, 10 pages, 2002.

[27] O. Sahin and N. Erina, "High-resolution and large dynamic range nanomechanical mapping in tapping-mode atomic force microscopy," Nanotechnology, vol. 19, no. 44, Article ID 445717, 2008.

[28] D. W. Chun, K. S. Hwang, K. Eom et al., "Detection of the $\mathrm{Au}$ thin-layer in the $\mathrm{Hz}$ per picogram regime based on the microcantilevers," Sensors and Actuators A, vol. 135, no. 2, pp. 857-862, 2007.

[29] N. Gitis, M. Vinogradov, I. Hermann, and S. Kuiry, "Comprehensive mechanical and tribological characterization of ultrathin films," MRS Proceedings, vol. 1049, 2008.

[30] A. F. Jankowski, "Vapor deposition and characterization of nanocrystalline nanolaminates," Surface and Coatings Technology, vol. 203, no. 5-7, pp. 484-489, 2008.

[31] A. F. Jankowski, "On eliminating deposition-induced amorphization of interfaces in refractory metal multilayer systems," Thin Solid Films, vol. 220, no. 1-2, pp. 166-171, 1992.

[32] A. F. Jankowski, J. P. Hayes, T. E. Felter, C. Evans, and A. J. Nelson, "Sputter deposition of silicon-oxide coatings", Thin Solid Films, vol. 420-421, pp. 43-46, 2002.

[33] A. F. Jankowski, M. A. Wall, A. W. Van Buuren, T. G. Nieh, and J. Wadsworth, "From nanocrystalline to amorphous structure in beryllium-based coatings," Acta Materialia, vol. 50, no. 19, pp. 4791-4800, 2002.

[34] A. F. Jankowski, C. K. Saw, C. C. Walton, J. P. Hayes, and J. Nilsen, "Boron-carbide barrier layers in scandium-silicon multilayers," Thin Solid Films, vol. 469-470, pp. 372-376, 2004.

[35] A. F. Jankowski, J. P. Hayes, and C. K. Saw, "Dimensional attributes in enhanced hardness of nanocrystalline Ta-V nanolaminates," Philosophical Magazine, vol. 87, no. 16, pp. 2323-2334, 2007.

[36] A. F. Jankowski and M. A. Wall, "Transmission electron microscopy of Ni/Ti neutron mirrors," Thin Solid Films, vol. 181, no. 1-2, pp. 305-312, 1989.

[37] A. F. Jankowski and M. A. Wall, "Synthesis and characterization of nanophase face-centered-cubic titanium," Nanostructured Materials, vol. 7, no. 1-2, pp. 89-94, 1996.

[38] H.S. T. Ahmed, Use of dynamic test methods to reveal mechanical properties of nanomaterials [Ph.D. thesis], Texas Tech University, 2010.

[39] J. F. Nye, Physical Properties of Crystals, Oxford Press, Oxford, UK, 1960.

[40] J. R. Neighbours and G. A. Alers, "Elastic constants of silver and gold," Physical Review, vol. 111, no. 3, pp. 707-712, 1958.

[41] G. A. Alers, "Elastic moduli of vanadium," Physical Review, vol. 119, no. 5, pp. 1532-1535, 1960.

[42] F. H. Featherston and J. R. Neighbours, "Elastic constants of tantalum, tungsten, and molybdenum," Physical Review, vol. 130, no. 4, pp. 1324-1333, 1963.

[43] B. T. Bernstein, "Elastic constants of synthetic sapphire at $27^{\circ}$," Journal of Applied Physics, vol. 34, no. 1, pp. 169-172, 1963.

[44] H. M. Trent, D. E. Stone, and L. A. Beaubien, "Elastic constants, hardness, strength, elastic limits, and diffusion coefficients of solids," in American Institute of Physics Handbook, Section 2, pp. 49-59, McGraw Hill, New York, NY, USA, 1972.

[45] H. P. R. Frederikse, "Elastic Constants of Single Crystals," in Handbook of Chemistry and Physics, D. Lide, Ed., section 12, pp. 33-38, CRC Press Taylor and Francis, Boca Raton, Fla, USA, 88th edition, 2008. 
[46] J. Hay, Application Notes 5990-4853EN (Agilent Technology), 2009, .

[47] Y. Yamada-Takamura, E. Shimono, and T. Toshida, "Nanoindentation characterization of cBN films deposited from vapor phase," in Proceedings of the 14th International Symposium on Plasma Chemistry (ISPC '14), M. Hrabovsky, Ed., vol. 3, pp. 1629-1634, Prague, Czech Republic.

[48] J. Y. Rho and G. M. Pharr, "Nanoindentation testing of bone," in Mechanical Testing of Bone and the Bone-Implant Interface, Y. H. An and R. A. Draughn, Eds., chapter 17, pp. 257-269, CRC Press, New York, NY, USA, 2000.

[49] K. L. Johnson, K. Kendall, and A. D. Roberts, "Surface energy and the contact of elastic solids," in Proceedings of the Royal Society A, vol. 324, pp. 301-313, 1971.

[50] Y.-P. Zhao, X. Shi, and W. J. Li, "Effect of work of adhesion on nanoindentation," Reviews on Advanced Materials Science, vol. 5, no. 4, pp. 348-353, 2003.

[51] B. V. Derjaguin, V. M. Muller, and Y. P. Toporov, "Effect of contact deformations on the adhesion of particles," Journal of Colloid And Interface Science, vol. 53, no. 2, pp. 314-326, 1975.

[52] J. Drelich, "Adhesion forces measured between particles and substrates with nano-roughness," Minerals and Metallurgical Processing, vol. 23, no. 4, pp. 226-232, 2006.

[53] M. M. McCann, Nanoindentation of gold single crystals [Ph.D. thesis], Virginia Polytechnic Institute and State University, 2004. 

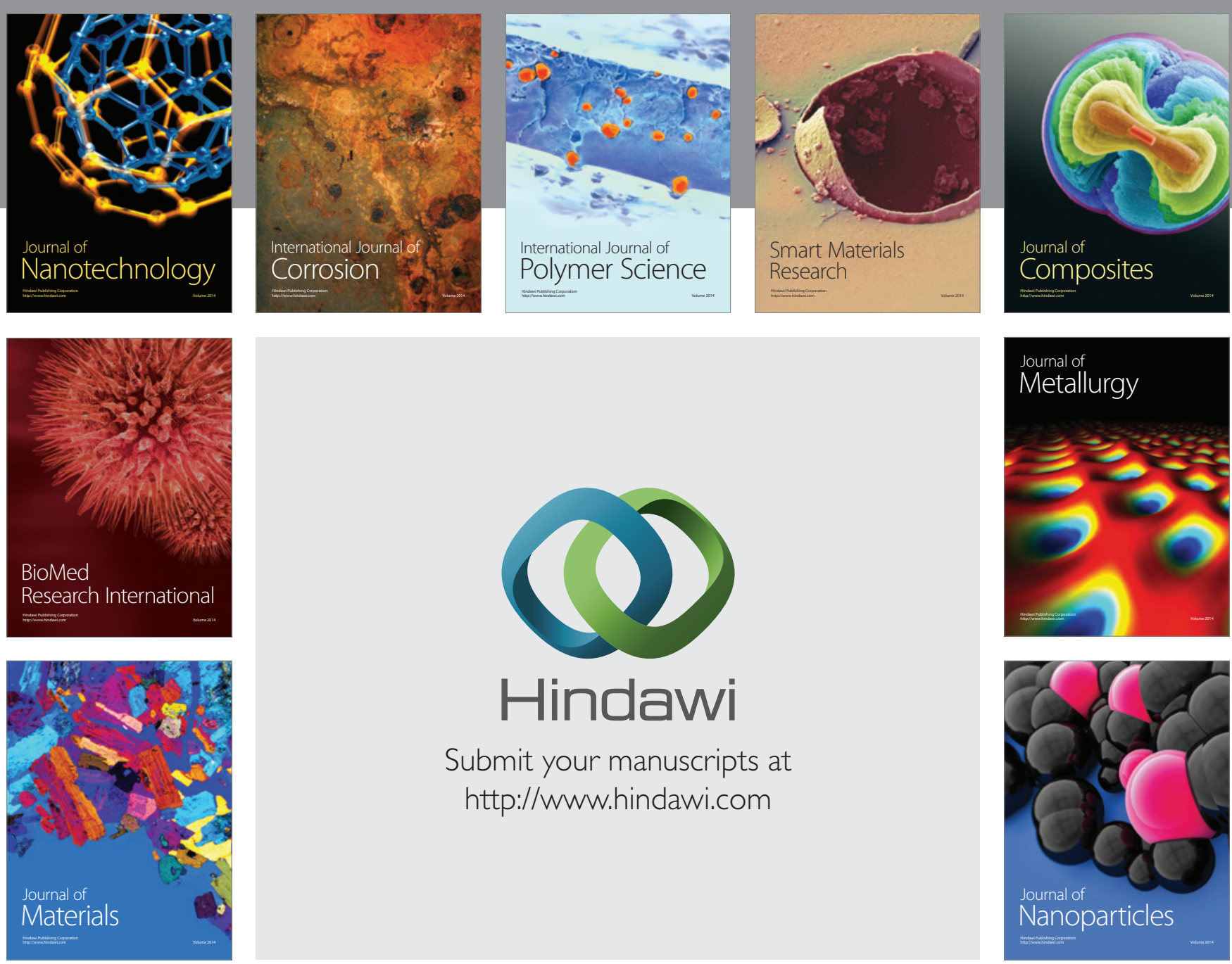

Submit your manuscripts at http://www.hindawi.com
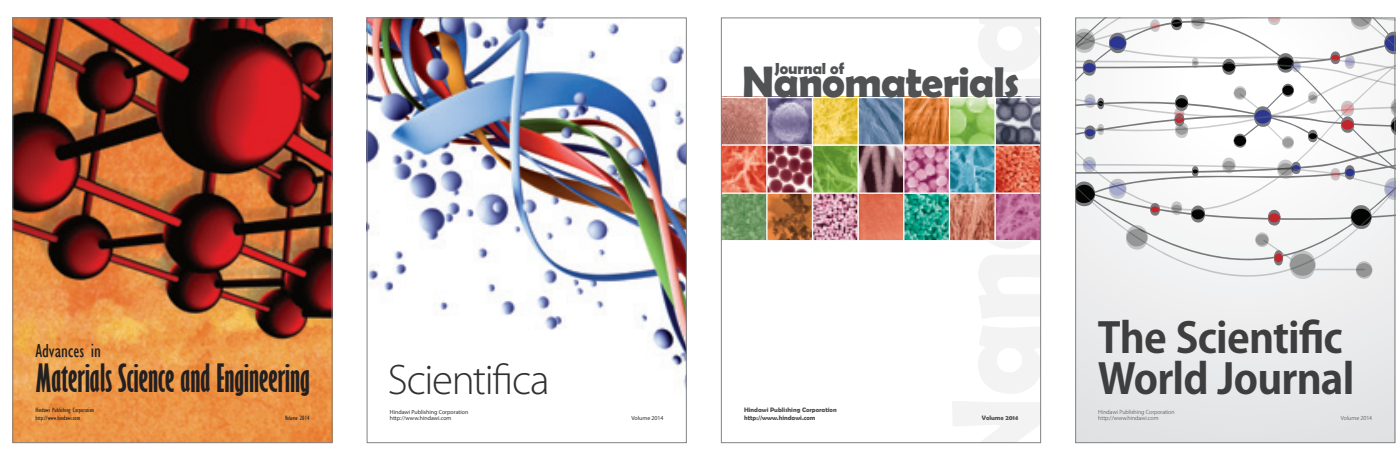

\section{The Scientific World Journal}
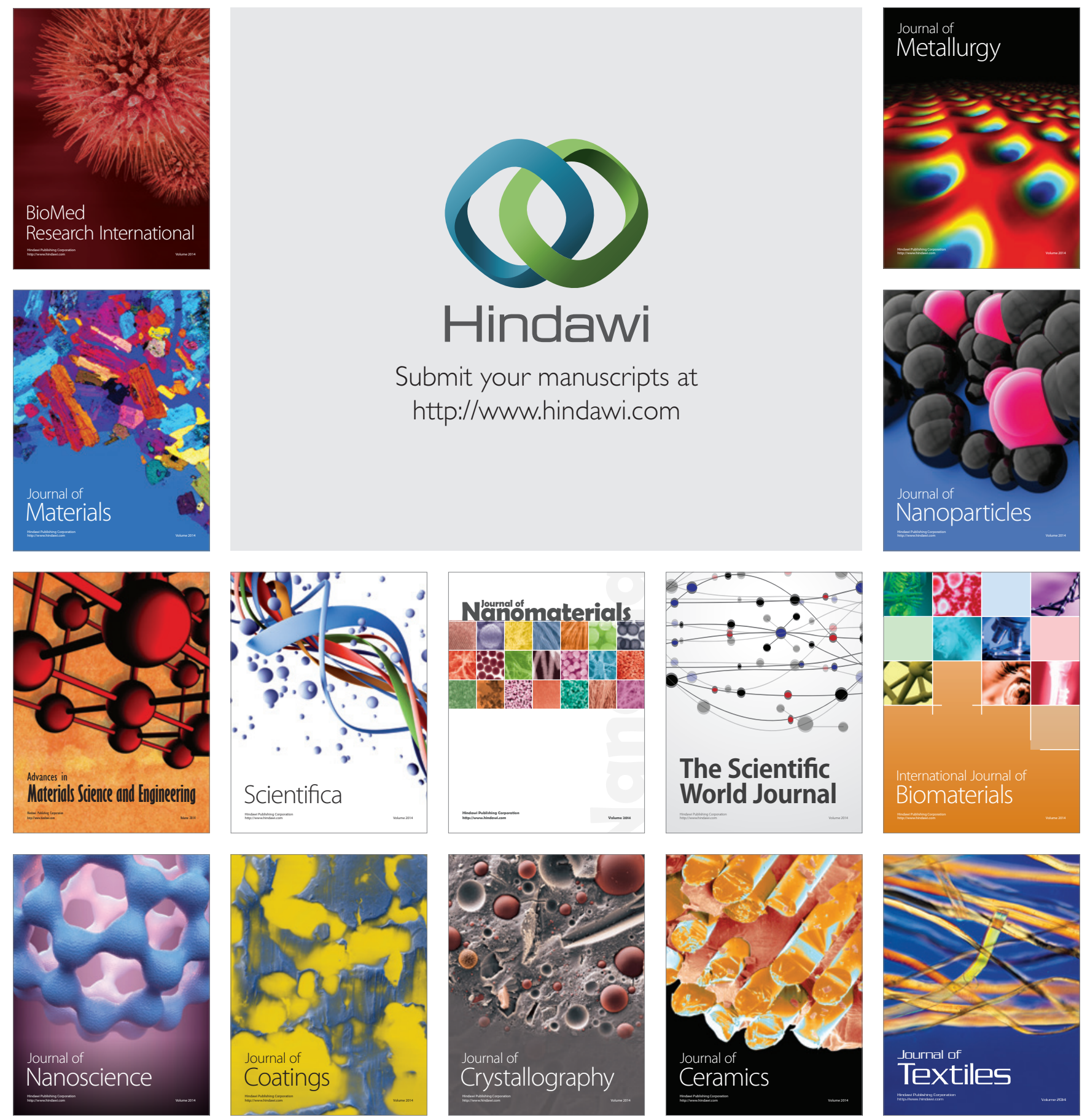